\title{
On Cyclic Codes of Odd Lengths from the Stable Variety of Regular Cayley Graphs
}

\author{
Chun P.B ${ }^{1, *}$, Ibrahim A.A ${ }^{2}$, Kamoh N.M ${ }^{3}$ \\ ${ }^{1}$ Department of Mathematics, Plateau State University, Jos, Nigeria \\ ${ }^{2}$ Department of Mathematics, Usmanu Danfodiyo University, Sokoto, Nigeria \\ ${ }^{3}$ Department of Mathematics, Bingham University, Karu, Abuja, Nigeria
}

Copyright $\mathrm{O} 2018$ by authors, all rights reserved. Authors agree that this article remains permanently open access under the terms of the Creative Commons Attribution License 4.0 International License

\begin{abstract}
The use of the adjacency matrix of a graph as a generator matrix for some classes of binary codes had been reported and studied. This paper concerns the utilization of the stable variety of Cayley regular graphs of odd order for efficient interconnection networks as studied, in the area of Codes Generation and Analysis. The Use of some succession scheme in the construction of a stable variety of the Cayley regular graph had been considered. We shall enumerate the adjacency matrices of the regular Cayley graphs so constructed which are of odd order $(2 m+1)$, for $m \geq 3$ as in [1]. Next, we would show that the Matrices are cyclic and can be used in the generation of cyclic codes of odd lengths.
\end{abstract}

Keywords Cayley Graph, Cyclic Codes, Cyclic Shift, Degree of a Graph, Non-Negative Matrix, Regular Graph, Symmetric Matrix

\section{Introduction}

Graph theory, which is generally concerned with the formulation of interconnection networks, has found applications in many real life situations that include, graph theoretic properties of some special permutation sub words, combinatorics of succession and algebraic construction of efficient broadcast networks. The Cayley graph is one of the important classes of graphs that have been used for some design problems especially involving the degree/diameter problems for finding largest possible networks satisfying some bounds on maximum node degree and diameter [1]. In mathematics, a Cayley graph is a graph that encodes the abstract structure of a group. The Cayley graph was first considered for finite groups by Arthur Cayley in 1872 [2]. Max Dehn in his unpublished lectures on group theory from 1909 reintroduced Cayley graphs under the name Gruppenbild (Group Diagram) which led to the geometric group theory of today. His most important application was the solution of the word problem for the fundamental group of surfaces with genus $n \geq 2$, which is equivalent to the topological problem of deciding which closed surface contract to a point [6]. Ibrahim \& Audu (2010) considered the use of some succession scheme in the construction of a variety of Cayley graphs that could be used as interconnection networks.

On the other hand, coding theory, which had been described by many authors as 'the mathematical theory of communication', conventionally defined as the study of the methods for efficient and accurate, storage and transfer of information from one place to another. The theory had found relationship with some other important fields to include, design theory, graph theory, lattices theory, group theory and so on. The class of cyclic codes is considered one of the most important classes of binary codes due to their easy encoding and decoding schemes. The natural requirement that a cyclic shift $(\pi)$ in the bits of a codeword of a cyclic code $C$ is still a codeword of $C$ makes their encoding and decoding easy. A code $C$ is said to be cyclic, if a cyclic shift of each of its codeword results to another codeword also in $C$. The report by Williem (2011) that the adjacency matrix of a graph can be interpreted as the generator matrix of a binary code [3], pointed another direction in the relationship between the two areas. Some small classes of linear and cyclic codes from the adjacency matrices of some Eulerian graphs due to the $(132) /(123)$-avoiding patterns of Audu and Aminu (AUNU) permutations [4,5].

In this paper, we enumerate the adjacency matrices of the stable variety of Cayley graphs constructed by Ibrahim \& Audu (2010) using some succession schemes. We shall then use the matrices so enumerated which are cyclic to span a cyclic class of codes of odd lengths and whose odd length property is a direct consequence of the odd order of this variety of Cayley graphs.

\section{Basic Concepts}

To make this communication self-explanatory, some 
basic and important concepts are highlighted. Some of which includes;

Cyclic Codes: A linear code $C$ of length $n$ over $F$ is cyclic if any cyclic shift of a code word is again a code word, i.e if $\left(a_{0}, a_{1}, \ldots a_{n-1}\right)$ is in $C$, then so is the code word $\left(a_{n-1}, a_{0}, a_{1}, \ldots a_{n-2}\right)$.

AUNU Patterns: The term AUNU pattern refers to a pairing scheme involving pairs of numbers associated by some precedence relation (Ibrahim, 2004a) and (Ibrahim, 2005) as cited in (Buhari, 2014).

Permutation Patterns: An arrangement of the objects $1,2, \ldots, n$ is a sequence consisting of these objects arranged in any order. When in addition, a particular order of arrangement is desired, such an arrangement becomes an ordered arrangement govern by a pattern $\sigma$ and each such permutation $\sigma \in S_{n}$ naturally results into a certain arrangement of $1,2, \ldots, n$ given by $s(1) s(2) \ldots s(n)$, which is called the arrangement associated with a permutation pattern $\sigma$ of points of a non-empty set $W=\{1,2, \ldots, n\}$.

\section{A Graph}

A graph consist of a finite set of vertices together with a set of edges, where each edge is a subset of the vertex set of cadinality two (2). If $p$ is a vertex of a graph $\Gamma$, the valency of $p$ is the number of edges containing $p$ (equivalently, the number $|\Gamma(p)|$ of vertices adjacent to $p$ ). If every vertex has the same valency, the graph is termed regular.

\section{Remark}

The diameter of a graph is the largest value $d$ defined by $d(p, q)=$ length of the shortest path containing $p$ and $q$.

\section{Symmetric Matrix}

A square matrix $A$, is symmetric if $A^{T}=A$, where $A^{T}$ denotes the transpose of $A$.

i.e the ijth element $a_{i j}$ is equal to the jith element. Moreover, the matrix $A$ is aid to skew-symmetric if $A^{T}=-A$ i.e the ijth element $a_{i j}$ is -1 times the jith element. i.e $a_{i j}=-a_{i j}$

\section{Generator Matrix}

A generator matrix $G$ for a linear code $C$ is a $k \times n$ matrix for which the rows are a basis for $C$. If $G$ is a generator matrix for $C$, then $C=\left\{a G \mid a \in F^{k}\right\} . G$ is said to be in standard form(often called the Reduce Echelon form) if $G=\left(I_{k} \mid X\right)$ where $I_{k}$ is the $k \times k$ identity matrix.

Remark: If $G$ is in standard form, then the first $k$ symbols or bits of a code word are called information symbols.

\section{Methodology}

\section{Formulation of the 2-Stable Degree/Diameter Network Model}

Here, we present a step by step procedure of the succession scheme for the construction of this variety of Cayley graphs as in [1].

(a) Let $n$ be the number of vertices such that $n$ is odd and $n \geq 5$

(b) Formulate a 2-stage succession scheme by combining pairs of elements (nodes)as element and $1^{\text {st }}$ successor in the second stage and as element and $2^{\text {nd }}$ successor in the second stage as follows;

\section{i) Element and $1^{\text {st }}$ Successor}

$$
S_{1 s t}=i, i+1, \ldots, n, i
$$

where $i$ is a fixed value representing the first of the elements in the sequence of points of $\Omega=\{1,2, \ldots, n\}$ permuted in cyclic form so that the number $1,2, \ldots, n$ represent the corresponding positions in ascending order. The form (1) is therefore a cycle

$$
(i, i+1, \ldots, n)
$$

\section{ii) Element and 2nd Successor}

$$
S_{2 n d}=i, i+2, \ldots, n-1, i
$$

Equivalently, (3) generates the following circle

$$
(i, i+2, \ldots, n-1, i
$$

It is evident that (3) and (4) are the required generators of the cyclic group from which the required Cayley graph can be constructed using a simple algorithm (see[7]) and we therefore have the following,

$\left\{\right.$ Form circuits $C_{1, i}, C_{2, i}$ by converting vertices $v_{i_{1}}, v_{i_{2}}$ and $\left.v_{j_{1}}, v_{j_{2}}\right\}$

1. Set $i_{1}, i_{2}=0$

2. Repeat 1 if $j_{n}=i_{n}+n$, do the following: Connect $v_{i_{n}}$ and $v_{j_{n}}$ as tail and head respectively.

3. Until $v_{j_{n}}=v_{0}$

4. If $v_{j_{n}}=v_{0}$ then go to step 2

5. End 
Based on the foregoing, a more stable design, which is proposed for interconnection networks, is also based upon the desire for optimality in respect of Degree/Diameter problems. These are Cayley sub graphs of the ones reported earlier with additional property of being defined for all odd number of vertices. Thus the order of such a sub graph is:

$$
N=|G|=2 m+1, m \geq 3
$$

We consider the regular Cayley graphs of orders 7 and 9 as in [1];

\section{Enumeration of the Adjacency Matrices of the Cayley Graphs}

The adjacency matrices for the illustrated Cayley regular graphs of orders 7 and 9 i.e for $m=3$ and $m=4$ are as depicted in the matrices $A$ and $B$ below respectively.

$$
A=\left[\begin{array}{lllllll}
0 & 1 & 1 & 0 & 0 & 1 & 1 \\
1 & 0 & 1 & 1 & 0 & 0 & 1 \\
1 & 1 & 0 & 1 & 1 & 0 & 0 \\
0 & 1 & 1 & 0 & 1 & 1 & 0 \\
0 & 0 & 1 & 1 & 0 & 1 & 1 \\
1 & 0 & 0 & 1 & 1 & 0 & 1 \\
1 & 1 & 0 & 0 & 1 & 1 & 0
\end{array}\right]
$$

Matrix A: Adjacency Matrix for the Stable Variety of Cayley Regular graph of order 7

$$
B=\left[\begin{array}{lllllllll}
0 & 1 & 1 & 0 & 0 & 0 & 0 & 1 & 1 \\
1 & 0 & 1 & 1 & 0 & 0 & 0 & 0 & 1 \\
1 & 1 & 0 & 1 & 1 & 0 & 0 & 0 & 0 \\
0 & 1 & 1 & 0 & 1 & 1 & 0 & 0 & 0 \\
0 & 0 & 1 & 1 & 0 & 1 & 1 & 0 & 0 \\
0 & 0 & 0 & 1 & 1 & 0 & 1 & 1 & 0 \\
0 & 0 & 0 & 0 & 1 & 1 & 0 & 1 & 1 \\
1 & 0 & 0 & 0 & 0 & 1 & 1 & 0 & 1 \\
1 & 1 & 0 & 0 & 0 & 0 & 1 & 1 & 0
\end{array}\right]
$$

Matrix B: Adjacency Matrix for the Stable Variety of Cayley Regular graph of order 9

The two Matrices $A$ and $B$ above are the adjacency Matrices of the stable variety of Cayley regular graphs of odd $(2 m+1)$ orders 7 and 9 for $m=3$ and $m=4$ respectively.

\section{Discussion of Results}

The two matrices $A$ and $B$ enumerated above are clearly seen to be circular, symmetric, has zero diagonals and are non-negative, nonzero Matrices [8]. Moreover, they are equally reflexive along their leading diagonals.

\section{Remark}

We note also that the matrix $G^{1}$ as in [5], which generated a linear cyclic code $C^{1}$ with 32 code words and an odd length of $n=5$ is also cyclic. Furthermore, if the elements on the upper triangular part of $G^{1}$ are complemented, the matrix $G^{1}$ is equally clearly seen to be reflexive as in the case of $A$ and $B$. Thus, an isomorphism is possible between the two classes of matrices.

\section{Conclusion}

Based on the forgoing, we may generalize that, the matrices $A$ and $B$, after some matrix row operations are generator matrices for some class of cyclic codes of odd lengths $n$ and has sizes $M=2^{n}$, where $M \in \mathbb{N}$.

\section{REFERENCES}

[1] A Ibrahim, and M Audu, (2005) On Stable Variety of Cayley Graphs for Efficient Interconnection Networks. African Journal of Natural Sciences Vol.8: 79-84

[2] A Cayley, (1878) The Theory of Groups: Graphical Representation. American Journal of Mathematics 1(2): 174-176 doi:10.2307/2369306.

[3] Willem, H. H (2011) Matrices for graphs, Designs and Codes, (NATO Science for Peace and security, series 29 Information Security), Coding theory and Related Combinatorics, IOS press, 2011

[4] B Chun, A Ibrahim and A Garba, (2016) Algebraic theoretic properties of the avoiding class of AUNU permutation patterns: Application in the generation and analysis of linear codes. International Organization for Scientific Research (IOSR), Journal of Mathematics 12(1) pp 1-3.

[5] B Chun, A Ibrahim and A, Garba, (2016). Algebraic Theoretic Properties of the Non-associative Class of (132)-Avoiding Patterns of AUNU Permutations: Applications in the Generation and Analysis of a General Cyclic Code. Computer Science and Information Technology, 4, 45-47. doi: 10.13189/csit.2016.040201.

[6] A Ibrahim, (2005) On the Combinatorics of Succession in a 5-Element sample. Abacus Journal of Mathematical Association of Nigeria Vol 32 No 4B:410-415.

[7] M Ibrahim, A Ibrahim, and A Yakubu, (2012) Algebraic theoretic properties of the (132)-Avoiding class of Aunu patterns application in Eulerian graphs, Journal of Science and Technology Research, 2012.

[8] F Shmuel, (2015) Matrices: Algebra, Analysis and Applications. World Scientific publishing Co. Pte. Ltd, Singapore pp. 399.

[9] Ding, C.(1962) Codes from Difference sets World Scientific Publishing Co. Pte. Ltd 5 Toh Tuck Link Singapore. 